FACULDADE DE CIÊNCIAS ECONÔMICAS DA UFRGS Análise
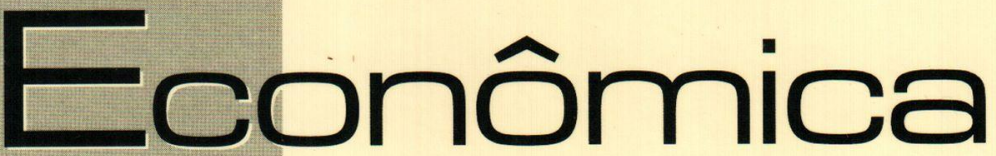

FLUTUACÃO CAMBIAL E METAS INFLACIONÁRIAS EM

ECONOMIAS EMERGENTES

OTAVIANO CANUTO E MárCIO HOLLAND

ATEORIA DE FORMACĆOO DE PRECOS E A TEORIA DOS PREÇOS DE

PRODUCAO

LOIZALBERTO ESTEVES

AECONOMIA EVOLUCIONISTA: UM CAPITULOSISTEMMICO DA

TEORIA ECONOMICA?

HUGOE. A. DA GAMACERQUEIRA

RELAÇ̃O INFLAÇÃO-PRODUTO NO BRASIL NO PERIODO PÓS-

REAL

MARCOSC. HOLANDA

DIVIIDA PÚBLICAMOBILLÍRIA FEDERAL BRASILEIRA: HISTÓRIA RECENTE E PERSPECTIVAS EXPLOSIVAS

ROGÉRIO MEURER E ROBERT WAYNE SAMOHYI

A CEPAL EA INTEGRACÃO REGIONAL LATINO-AMERICANA JACQUELINE A. HERNANDEZ HAFFNER

POPPER, HAYEK EA (IM)POSSIBILIDADE DE PREDIÇŌES ESPECIFICAS EM CIEENCIAS SOCIAIS

BRENA PAULA MAGNO FERNANDEZ

DINÂMICA RECENTE DO PROCESSO DE INCUBACG̃O DE

EMPRESAS DE BASE TECNOLOGICA NO BRASIL

EDUARDO GONÇALVES

TRIBUTACĀO COM SACRIF́CIO EQUUITATIVO: O CASO DO IMPOSTO DE RENDA PESSOA FISICA

STEFANO FLORISSI E EDUARDO PONTUAL RIBEIRO

A NOVATEORIA DO IMPÉRIOE AS VELHAS TEORIAS DO

IMPERIALISMO

GENTIL CORAZZA

Ô ABREALAS - A NOVA INSERÇÃO DO BRASIL NA ECONOMIA MUNDIAL

EMLLLANO LUIS KLEN

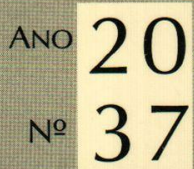

Março, 2002 
UNIVERSIDADE FEDERAL DO RIO GRANDE DO SUL

Reitora: Profa. Wrana Maria Panizzi

FACULDADE DE CIÊNCIAS ECONÔMICAS

Diretor: Prof. Pedro Cézar Dutra Fonseca

CENTRO DE. ESTUDOS E PESQUISAS ECONÔMICAS

Diretor: Prof. Gentil Corazza

DEPARTAMENTO DE CIÊNCIAS ECONÔMICAS

Chefe: Prof. Luiz Alberto Oliveira Ribeiro de Miranda

DAPARTAMENTO DE CIÊNCIAS CONTÁBEIS E ATUARIAIS

Chefe: João Marcos Leão da Rocha

CURSO DE PÓS-GRADUAÇÃO EM ECONOMIA

Coordenador: Prof. Eduardo Pontual Ribeiro

CURSO DE PÓS-GRADUAÇÃO EM ECONOMIA RURAL

Coordenador: Prof Jalcione Almeida

CONSELHO EDITORIAL: Carlos G. A. Mielitz Netto (UFRGS), Eduardo A. Maldonado Filho (UFRGS), Eduardo P. Ribeiro (UFRGS), Eleutério F. S. Prado (USP), Eugênio Lagemann (UFRGS), Fernando Cardim de Carvalho (UFRJ), Fernando Ferrari Filho (UFRGS), Fernando de Holanda Barbosa (FGV/RJ), Flávio Vasconcellos Comim (UFRGS), Gentil Corazza (UFRGS), Giácomo Balbinotto Neto (UFRGS), Gustavo Franco (PUC/RJ), Jan A. Kregel (Università di Bologna e John Hopkins University), João Rogério Sanson (UFSC), Joaquim Pinto de Andrade (UnB), Jorge Paulo Araújo (UFRGS), Juan H. Moldau (USP), Marcelo S. Portugal (UFRGS), Maria Alice L.ahorgue (UFRGS), Paul Davidson (University of Tennessee), Paulo Dabdab Waquil (UFRGS), Pedro Cézar Dutra Fonseca (UFRGS), Philip Arestis (South Bank University), Roberto C. Moraes (UFRGS), Ronald Otto Hillbrecht (UFRGS), Sabino da Silva Porto Jr. (UFRGS), Stefano Florissi (UFRGS), Werner Baer (Univ, of Illinois at Urbana-Champaign).

COMISSÃO EDITORIAL: Eduardo Augusto Maldonado Filho, Fernando Ferrari Filho, Gentil Corazza, Marcelo Savino Portugal, Paulo Dabdab Waquil; Roberto Camps Moraes.

EDITOR: Fernando Ferrari Filho

EDITOR ADJUNTO: Gentil Corrazza

SECRETÁRIA: Vanessa Hoffmann de Quadros

REVISÃO DE TEXTOS: Vanete Ricacheski

FUNDADOR: Prof. Antônio Carlos Santos Rosa

Os materiais publicados na revista Análise Econômica são da exclusiva responsabilidade dos autores. É permitida a reprodução total ou parcial dos trabalhos, desde que seja citada a fonte. Aceita-se permuta com revistas congêneres. Aceitam-se, também, livros para divulgação, elaboração de resenhas e recensões. Toda correspondência, material para publicação (vide normas na terceira capa), assinaturas e permutas devem ser dirigidos ao seguinte destinatário:

PROF FERNANDO FERRARI FILHO

Revista Análise Econômica - Av. João Pessoa, 52 CEP 90040-000 PORTO ALEGRE - RS, BRASIL Telefones: (051) 316-3348 e 316-3440 - Fax: (051) 316-3990

E-mail: rae@vortex ufrgs br

Análise Econômica

Ano 20, n 37 , março, 2002 - Porto Alegre

Faculdade de Ciências Econômicas, UFRGS, 2000

Periodicidade semestral, março e setembro.

1. Teoria Econômica - Desenvolvimento Regional Economia Agrícola - Pesquisa Teórica e Aplicada -

Periódicos. I. Brasil.

Faculdade de Ciências Econômicas,

Universidade Federal do Rio Grande do Sul.

CDD 330.05

CDU 33 (81) (05) 


\title{
Tributação com Sacrifício Eqüitativo: O caso do Imposto de Renda Pessoa Física'
}

\author{
Stefano Florissi $i^{2}$ e Eduardo Pontual Ribeiro ${ }^{3}$
}

\begin{abstract}
Resumo: Este trabalho visa verificar a hipótese de que o Imposto de Renda Pessoa Física (IRPF) impõe um sacrifício eqüitativo sobre os contribuintes. Usando a metodologia desenvolvida por Young (1990), resultados empíricos ex-post confirmam tal hipótese para o IRPF durante a década de 1980.
\end{abstract}

Palavras-chave: Imposto de renda; sacrificio eqüitativo.

\begin{abstract}
One of the theoretical principles of taxation is that a tax should impose an equal burden on taxpayers. The objective of this work is to investigate empirically, whether this principle is followed by the personal income tax in Brazil. Our results indicate that the personal income tax in Brazil closely approximates an equal sacrifice tax.
\end{abstract}

Keywords: personal income tax, equal sacrifice tax.

\section{Introdução}

A Teoria da Tributação baseia-se em dois princípios básicos, o da neutralidade e o da eqüidade. Enquanto o primeiro mantém que os impostos devem distorcer ao mínimo a alocaçāo de recursos, o segundo determina que o ônus tributário deve ser distribuído de forma eqüitativa para toda a população (Silva, 1978). O princípio da eqüidade tem origem com Adam Smith em seu livro The Wealth of the Nations, tendo sido elaborado e elevado a estatura de paradigma por John Stuart Mill (1923). Nas palavras deste autor:

Equity of taxation, therefore, as a maxim of politics, means equality of sacrifice. It means apportioning the contribution of each person towards the expenses of

\footnotetext{
${ }^{1}$ Gostariamos de agradecer a Níton Araújo, Francisco Cribari Neto e Jane Leuthold por valiosos comentários, em particular ao Prof. Leuthold que chamou nossa atenção para o artigo de Young (1990). Quaisquer erros remanescentes são de nossa única responsabilidade. Os autores agradecem o apoio financeiro da CAPES e UFRR quando do inicio deste trabalho.

${ }^{2}$ Programa de Pós-graduação em Economia - UFRGS. E-mail: florissi@portoweb.com.br.

${ }^{3}$ Programa de Pós-graduação em Economia -UFRGS. E-mail: eribeiro@ufrgs.br.
} 
government so that he shall fell neither more nor less inconvenience from his share of payment that every other person experiences from his. (p. 804)

Dentro da escola neo-clássica, inconvenience é traduzido como perda de utilidade. Desta forma, o princípio do sacrifício significa perda de bem-estar e, seguindo a idéia original de Stuart Mill, consideramos sacrifício equitativo como perda de utilidade em termos absolutos ${ }^{4}$.

Nesta perspectiva, utilizando a metodologia desenvolvida por Young (1987,1990), busca-se verificar a hipótese de que o Imposto de Renda Pessoa Física (IRPF) no Brasil impōe um sacrifício equitativo absoluto aos contribuintes, utilizando-se dados agregados das declarações do IRPF no período 1979-1989.

Todavia, o estudo da hipótese de sacrifício eqüitativo não é livre de obstáculos. O primeiro, como referido em Silva (1978), é o fato de que tal análise baseia-se em comparações de utilidades individuais. Musgrave (1958) e Young (1990) contra-argumentam lançando mão da suposição de que a utilidade é medida igualmente entre todos os indivíduos através de uma 'norma social', que implica urna forma específica de uma função de utilidade. Esta 'norma social' seria uma unidade de medida comum que o governo utilizaria para construir a tabela para cálculo do imposto de renda. O segundo é que não são considerados os efeitos sobre a alocação de recursos. Embora este seja a motivação principal para a literatura sobre imposto ótimo, iniciada pelo Prêmio Nobel James Mirrlees (1971), Berliant e Gouveia (1993) apresentam argumentos que qualificam nossa análise, fazendo-a menos restritiva que parece à primeira vista. Estes autores estudaram a existência de um imposto, ou uma função imposto, que gera sacrifício eqüitativo e ainda mantém incentivos ao trabalho com preferências sobre lazer e renda. Os resultados indicam que tal função existe e é similar à empregada aqui, para uma função de utilidade aditivamente separável (additively sepparable) entre "lazer" e consumo (renda). Assim, podemos entender que os resultados abaixo consideram efeitos alocativos, sob a hipótese condicional que as preferências são separáveis aditivamente.

Este trabalho organiza-se da seguinte forma. Após a definição do problema a ser estudado e os objetivos do trabalho na introdução, pas-

\footnotetext{
${ }^{4}$ Ver Silva (1978), Musgrave (1959) e outros para análise de outros tipos de sacrifício. Dentre elas, considerar sacrificio proporcional não alteraria nossos resultados de forma qualitativa (Young 1990). Por outro lado, sacrifício marginal implica uma renda líquida após o pagamento do imposto igual para todos. Isto é inviável e insustentável e sempre considerado na literatura.
} 
samos ao desenvolvimento do modelo na segunda seção. A terceira seção apresenta os resultados empíricos e a última, as conclusões do trabalho.

\section{O Modelo}

O modelo usado é o desenvolvido por Young(1990) baseado em resultados teóricos de Young(1987). Neste último, o autor prova que, se existe sacrifício eqüitativo entre os contribuintes e se a medida de sacrifício é invariante com respeito à unidade de medida (renda nominal ou real, por exemplo), a função de utilidade empregada para medir o sacrifício tem de ser uma função de utilidade exponencial ou logarítmica, de modo a apresentar aversão ao risco proporcional constante. Além disso, para valores plausíveis do coeficiente de aversão ao risco proporcional ${ }^{5}$ e taxas de imposto menores de $100 \%$, tal tabela de contribuição do imposto de renda não é regressiva.

A progressividade do IRPF foi verificada por Dias Neto (1984) para o período 1975-1981. A progressividade do IRPF nos assegura um ponto de partida para o estudo empírico. O modelo de Young busca, basicamente, estimar o coeficiente de aversão ao risco relativo e, dado seu valor estimado, construir uma tabela de imposto que segue a hipótese de sacrifício equitativo que pode então ser comparada com a tabela existente do IRPF.

De acordo com o Teorema 2 de Young (1987), suponha-se que a função de utilidade, cujo único argumento é a renda, $x$, tem a forma:

$$
U(x)=-A(x)^{1-C}+B
$$

onde $A>0$ e $C$, o coeficiente de aversão ao risco proporcional é suposto constante e maior que um. $A$ e $B$ são constantes quaisquer. A hipótese de sacrifício equitativo implica que

$$
U(x)-U(x-t)=s,
$$

onde $t$ é o imposto ex post sobre o nível de renda $x$.

Dividindo ambos os lados de (2) por $t$ tem-se:

$$
\frac{U(x)-U(x-t)}{t}=\frac{s}{t} \text {. }
$$

s Para uma apresentação do coeficiente de aversão ao risco proporcional, ver, entre outros, Varian (1992), cap. 11. 
Pelo teorema do valor médio, o termo da esquerda em (3) é equivalente à derivada de $U(x)$ avaliada em um ponto $w \in[x-t, x]$. Sem perda de generalidade, considere-se $A=1$ e $B=0$. Tem-se, assim, que

$$
U^{\prime}(w)=(C-1) w^{-c}=[U(x)-U(x-t)] / t=s / t .
$$

Substituindo (1) em (4) e após alguns passos de álgebra, obtem-se

$$
\frac{w}{x}=\left[\frac{(C-1)(t / x)}{(1-t / x)^{1-C}-1}\right]^{1 / C} \text {. }
$$

Young mostra que, dado um valor constante de $t / x$, com diferentes valores de $C \in[1.1,3], w / x$ se mantém quase inalterado. Desta forma, pode-se tomar um $C$ qualquer neste intervalo e estimar $w / x$ com segurança. Por simplicidade, mantém-se $C=2$, que implica $w=\sqrt{x(x-t)}$. Substituindo em (4) e tomando logaritmos, tem-se que

$$
\ln t=C \ln \sqrt{x(x-t)}+\text { cons } \tan t e
$$

Este é o modelo de regressão que se irá estimar. Condicional à função de utilidade suposta, a hipótese de sacrifício eqüitativo será válida se se encontrar uma relação linear entre $Y=\ln t$ e $X=\frac{1}{2} \ln x(x-t)$, e o parâmetro da inclinação da reta será o valor estimado de $C$.

Estimado $C$, tem-se de verificar se a própria hipótese de sacrifício eqüitativo é válida. Para isso, primeiro, calcula-se $s=U(x)-U(x-t)$ usando os parâmetros estimados. Dado o valor obtido calcula-se uma tabela do imposto a pagar $\hat{t}$ usando (1) e os valores de $s$ e $C$ calculados, ou seja,

$$
\hat{t}=x-\left(x^{1-\hat{C}}+\hat{s}\right)^{\frac{1}{1-\hat{c}}}
$$

Se a diferença entre $t$ e $\hat{t}$ forem pequenas, a hipótese de sacrifício eqüitativo não será rejeitada, para a função de utilidade estimada.

\section{Resultados empíricos}

Nos resultados empíricos foram utilizados os dados agregados sobre renda e impostos pagos de vários números do Anuário Econômico Fiscal, referentes aos exercícios de 1979 a 1989. Usa-se como renda (x), a renda bruta calculada, isto é, o rendimento bruto menos as deduçōes 
das células A, B, C, D, E e F'. Subtrai-se da renda tributável as deduções, para levar em conta a renda mínima de subsistência, seguindo as idéias de Stuart Mill. Para o imposto $t$, utiliza-se o imposto devido calculado. Desta forma, abstraem-se as particularidades de cada indivíduo (abatimentos individuais) e as reduções por investimento, que têm como justificativa motivos outros que não o da eqüidade. Os dados são per capita, dentro de cada classe de rendimento bruto. A tabela 1 mostra os resultados das regressões usando MQO para a equação (6) para os anos de 1979 a 1989. A constante é deixada de lado por não ser relevante. Em todos os anos, as duas primeiras e a última classe de rendimento bruto não são consideradas, pois configuram-se claramente como outliers.

Tabela 1: Coeficiente de aversão ao risco proporcional (C)

\begin{tabular}{c|ccccc} 
Ano & $C$ & Erro padrão & $R^{2}$ & $s^{2}$ & $n$ \\
\hline 1979 & $2.3883^{\mathrm{a}}$ & 0.1025 & 0.9836 & 0.1015 & 11 \\
1980 & $2.3836^{\mathrm{a}}$ & 0.1047 & 0.9829 & 0.1068 & 11 \\
1981 & $2.3789^{\mathrm{a}}$ & 0.1079 & 0.9818 & 0.1152 & 11 \\
1982 & $2.3880^{\circ}$ & 0.1112 & 0.9808 & 0.1221 & 11 \\
1983 & $2.3830^{\circ}$ & 0.1038 & 0.9831 & 0.1068 & 11 \\
1984 & $2.3536^{\mathrm{a}}$ & 0.1013 & 0.9835 & 0.1053 & 11 \\
1985 & $2.3521^{\mathrm{a}}$ & 0.0949 & 0.9855 & 0.0934 & 11 \\
1986 & $2.3598^{\circ}$ & 0.1088 & 0.9812 & 0.1215 & 11 \\
1987 & $2.1570^{\circ}$ & 0.0571 & 0.9944 & 0.0351 & 10 \\
1988 & $2.1786^{\circ}$ & 0.0578 & 0.9943 & 0.0363 & 10 \\
1989 & $2.1350^{\circ}$ & 0.0633 & 0.9964 & 0.0084 & 6 \\
\hline
\end{tabular}

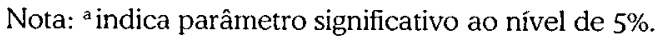

Como pode-se ver na tabela acima, o modelo estimado é satisfatório, dado o $\mathrm{R}^{2}$ e o valor do teste $t$ de Student para a inclinação da reta. $O$ valor de $C$ é constante ao longo dos períodos 79-86 e 87-89. No período de 1987-89, dadas mudanças significativas ocorridas no im-

\footnotetext{
' Para mais detalhes, ver SERF, Imposto de Renda Pessoa Física, vários números.
} 
posto de renda, o número de faixas de renda foi reduzido. Verificou-se, com isso, uma diminuição no valor do coeficiente.

O coeficiente $C$ está ligado positivamente ao grau de progressividade do imposto, o que pode ser visto através do resultado de Samuelson apud Berliant e Gouveia (1993), onde a progressividade do imposto para a função utilidade adotada no artigo pode ser visța pela condição (1 - $C) \ln (x /(x$ $-t))<0$. As reformas de 1987, que reduziram as alíquotas no espírito da reforma tributária de 1986 nos EUA, reduziram a progressividade do IRPF.

Vale a pena ressaltar que os valores do coeficiente de aversão relativa ao risco obtidos são maiores que os obtidos por Young (1990) para os Estados Unidos, mas próximos de 2 como esperado.

Usando os valores estimados de $C$ para cada ano, verifica-se quão boa é a aproximação da tabela do imposto de renda com aquela obtida usando C. Nas figuras 1 e 2, que representam os anos de 1983 e 1989 , respectivamente, vêem-se os resultados em termos de imposto médio, dentro de cada classe de rendimento bruto?

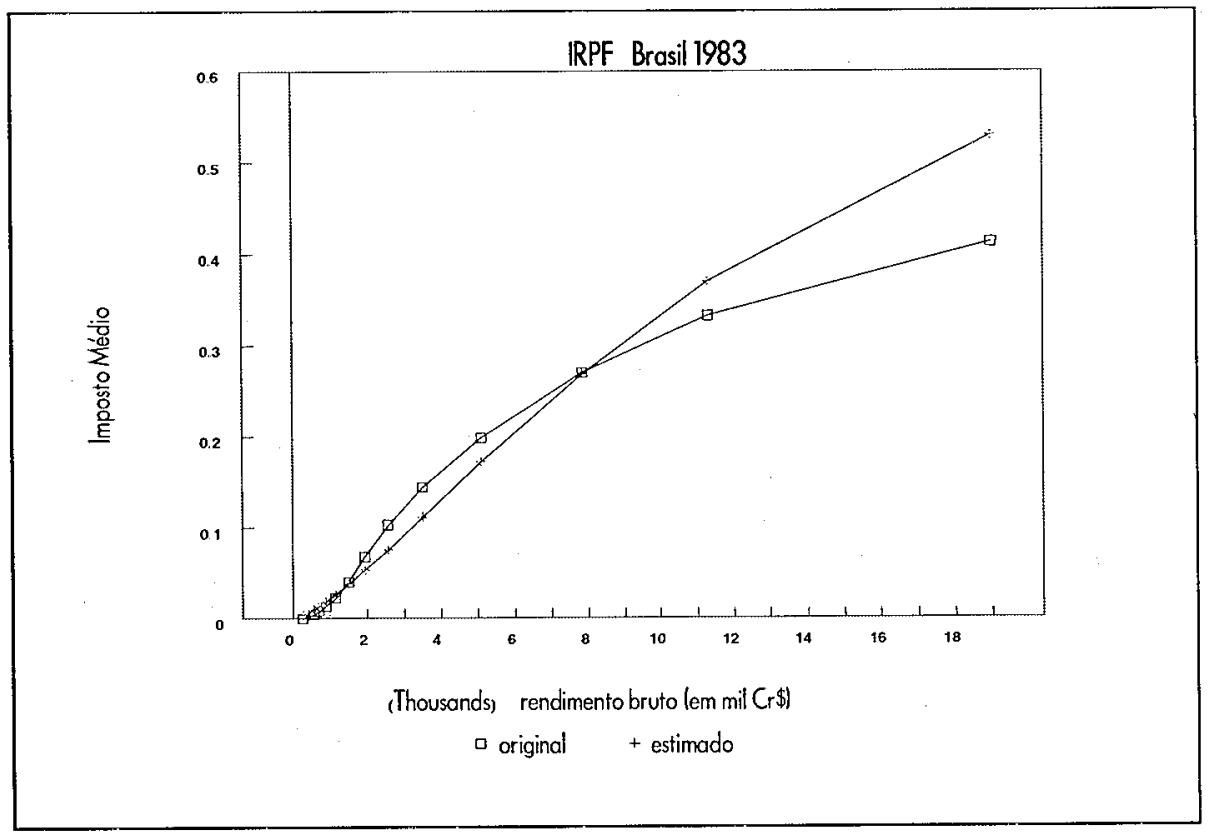

Figura 1: Imposto com sacrificio eq.: imposto médio

${ }^{7}$ Os gráficos para os outros anos não apresentam diferenças significativas, como esperado pelos 


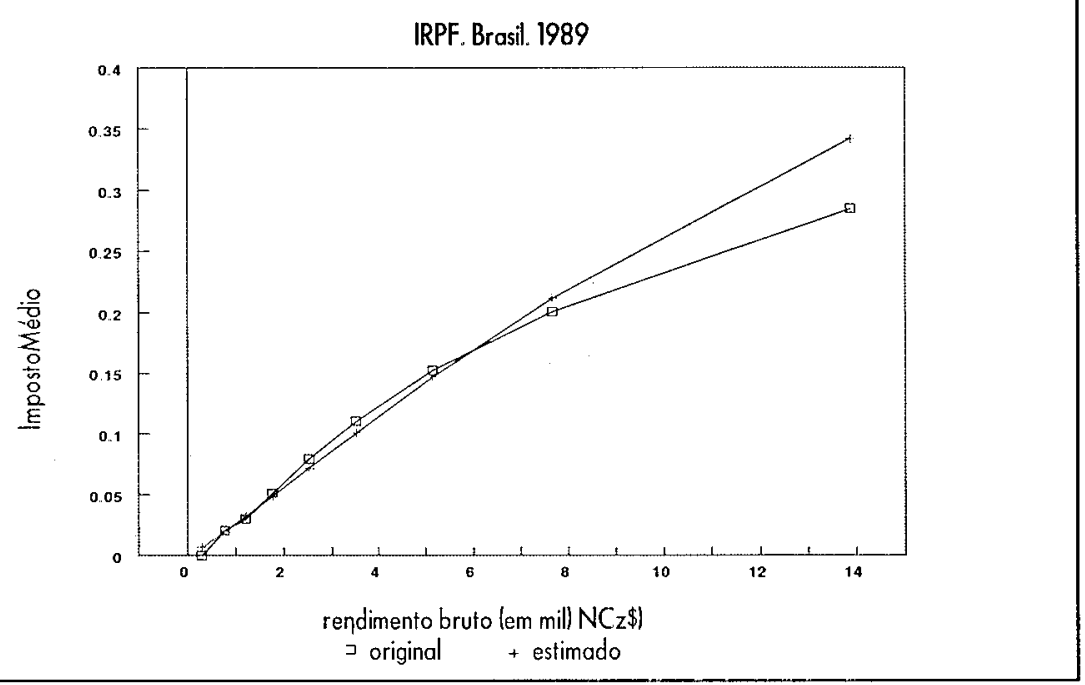

Figura 2: Imposto com sacrificio eq.: imposto médio

Vê-se que a aproximação é boa. Em realidade, para que o IRPF imponha sobre os contribuintes um sacrifício eqüitativo, strictu sensu, a carga média de imposto deve ser reduzida para os contribuintes nas classes médias de imposto e/ou deve-se aumentar o imposto calculado daqueles contribuintes nas classes mais baixas e altas de rendimento.

Por outro lado, entende-se que tal política provavelmente levará a uma tabela do imposto de renda menos progressiva, por aumentar a carga tributária dos contribuintes mais pobres, além de ter possíveis efeitos negativos sobre o esforço de trabalho, ou incentivo à evasão, daqueles nas classes mais altas.

\section{Conclusão}

Este trabalho buscou verificar a veracidade da hipótese que o IRPF no Brasil impõe um sacrificio eqüitativo a seus contribuintes, usando a metodologia de Young (1990) no período de 1979 a 1989.

Em suma, comprovou-se que o IRPF é aproximadamente um im. posto que impõe um sacrificio eqüitativo absoluto a seus contribuintes. As disparidades podem ser interpretadas pelo fato de que um nível de imposto médio menor que o previsto para as classes de renda bruta mais baixas é justificado para melhorar a progressividade do sistema, e que o Fisco busca diminuir o efeito negativo dos impostos na alocação 
de recursos nas classes de renda mais elevadas, como verificado em outros países. Lembrase que a hipótese de sacrifício absoluto, como estudado aqui, não leva em consideração tais efeitos, exceto sob a condição de preferências aditivamente separáveis entre horas de trabalho e renda.

Duas questões surgem deste trabalho. Primeiro, qual será a perda de bem-estar da sociedade se a tabela do IRPF seguir a hipótese de sacrifício eqüitativo em comparação com a atual tabela? E, segundo, quais os efeitos negativos, do ponto de vista da eficiência econômica, da atual tabela? O estudo destas questões seria conseqüência natural deste trabalho.

Para estudar estas questões, sugestões para estudos seriam o desenvolvimento de um teste empírico, nos moldes da metodologia usada, para o IRPF, seguindo os resultados de Berliant e Gouveia. Ou, por outro lado, a estimação da elasticidade-renda e salário da oferta de trabalho e com tais medir a perda de bem-estar derivada da existência do Imposto de Renda. Nesta última linha, sem verificar o nível de sacrifício, Ribeiro e Leuthold (1997) concluem que a perda de bem-estar associado ao IRPF para assalariados urbanos do setor privado é pequena, relativamente a países como EUA e Inglaterra.

\section{Referências bibliográficas}

BERLIAN, Marcus e GOUVEIA, Miguel. "Equal sacrifice and incentive compatible income taxation". Journal of Public Economics, v. 51: 219-240, Janeiro 1993.

BRASIL. Anuário Econômico Fiscal, Brasília: Gráfica da Receita Federal. (vários números).

DIAS NETO, João. Um Estudo do Grau de Progressividade do Imposto de Renda - Pessoa Física no Brasil: 1975-1981. Brasília: ESAF, 1984

MILL, John Stuart. Principles of Political Economy, new impression. London: Longmans Green, 1923.

MIRRLEES, James. An exploration in the theory of optimum income taxation. Review of Economic Studies, v. 38, 1: 175-208, 1971.

MUSGRAVE, Robert M. The Theory of Public Finance. New York: McGraw-Hill, 1959.

RIBEIRO, Eduardo. P. e LEUTHOLD, Jane. Preference structures and the excess burden of income taxes in Brazil. Center for International Business and Economic 
Research Discussion Paper 97-101, University of Illinois at Urbana-Champaign, 1997.

SERF. Imposto de Renda Pessoa Fissica. Brasília: Gráfica da Receita Federal, (vários números).

SILVA, Fernando R. Finanças Públicas. São Paulo: Atlas,1978.

VARIAN, Hal R. Microeconomic Analysis, 3a. edição. New York: Norton, 1992.

YOUNG, H. Peyton. "Progressive taxation and the equal sacrifice principle". Journal of Public Economics, 32:204-14, January, 1987.

. "Equal sacrifice and progressive taxation". American Economic Review, 80:253-66, 1990. 